\title{
LONG TURNS, INP'S AND INDICES FOR FREE GROUP AUTOMORPHISMS
}

\author{
THIERRY COULBOIS AND MARTIN LUSTIG
}

\begin{abstract}
The goal of this paper is to introduce a new tool, called long turns, which is a useful addition to the train track technology for automorphisms of free groups, in that it allows one to control periodic INPs in a train track map and hence the index of the induced automorphism.
\end{abstract}

\section{INTRODUCTION}

Automorphisms of a non-abelian free group $F_{N}$ of finite rank $N \geq 2$ are the focal point of many interesting recent research efforts. The most important class of such automorphisms are the ones that are irreducible with irreducible powers (iwip), also called fully irreducible (see §2). Such automorphisms can always be represented by a map $f: \Gamma \rightarrow \Gamma$, where $\Gamma$ is a graph equipped with a marking isomorphism $F_{N} \stackrel{\cong}{\longrightarrow} \pi_{1}(\Gamma)$, and $f$ has the train track property: the map $f$ defines a gate structure $\mathbf{G}=\mathbf{G}(f)$ on $\Gamma$, i.e. a partition of the edge germs at every vertex into gates, which is preserved by $f$ in that $f$ maps any G-legal path to a G-legal image path. Here a path $\gamma$ in $\Gamma$ is $\mathbf{G}$-legal if at any vertex it never enters and exits through the same gate.

In this paper we present a new tool, called long turns, which we use to define in $\S 5$ below legalizing train track morphisms $g: \Gamma \rightarrow \Gamma^{\prime}$ (with respect to fixed gate structures $\mathbf{G}$ on $\Gamma$ and $\mathbf{G}^{\prime}$ on $\Gamma^{\prime}$ ). A construction device for such legalizing train track morphisms is given in Section 7 .

Theorem 1.1. Let $f: \Gamma \rightarrow \Gamma$ be a train track map which represents an iwip automorphism of $F_{N}$ and has positive transition matrix $M(f)$. Let $g: \Gamma \rightarrow \Gamma$ be a legalizing train track morphism with respect to the gate structure $\mathbf{G}(f)$, and assume that $g$ induces an automorphism on $\pi_{1}(\Gamma)$ and is gate-stable (see Definition 3.11). Then:

(1) The map $f \circ g: \Gamma \rightarrow \Gamma$ is a train track representative of an iwip automorphism $\varphi \in \operatorname{Out}\left(F_{N}\right)$.

1991 Mathematics Subject Classification. 20E05, 20E08, 20F65, 57R30.

Key words and phrases. Free group automorphisms, Train tracks, Nielsen path, Index. 
(2) There is no periodic INP in $\Gamma$ for the train track map $f \circ g$. In particular there are no non-trivial $(f \circ g)$-periodic conjugacy classes in $F_{N}$.

(3) The stable index list for $\varphi$ is given by the gate index list for $f$.

To explain the last statement of this theorem we recall from [9] and [18] that for every automorphism $\Phi \in \operatorname{Aut}\left(F_{N}\right)$ with no periodic $w \in F_{N} \backslash\{1\}$ the induced action of a suitable positive power of $\Phi$ on $\partial F_{N}$ is particularly simple: There are finitely many attractors and finitely many repellers on $\partial F_{N}$, and every other orbit accumulates positively onto one of the attractors and negatively onto one of the repellers. The index of $\Phi$, as defined in [9], is in this special case equal to

$$
\operatorname{ind}(\Phi):=\frac{a(\Phi)}{2}-1
$$

where $a(\Phi)$ denotes the number of attractors of the $\Phi$-action on $\partial F_{N}$.

For any given $\varphi \in \operatorname{Out}\left(F_{N}\right)$ it has been shown in [9] that up to isogredience (= conjugation by inner automorphisms), there are only finitely many lifts $\Phi_{k} \in \operatorname{Aut}\left(F_{N}\right)$ of $\varphi$ which satisfy $\operatorname{ind}\left(\Phi_{k}\right)>0$. Those indices form the index list of $\varphi$ (which is defined up to permutation and typically given in decreasing order). Through replacing $\varphi$ by a suitable positive power $\varphi^{t}$ the number of terms and also their values in the index list may increase, but eventually it becomes stable (with respect to passing to further powers, see [9] or Section 8 below): this is called the stable index list of $\varphi$. It is an important invariant of the conjugacy class of $\varphi$ in $\operatorname{Out}\left(F_{N}\right)$ with interesting structural consequences (see [5]). The main result of [9] states that for the stable index of any $\varphi \in \operatorname{Out}\left(F_{N}\right)$, i.e. the sum of the values $\operatorname{ind}\left(\Phi_{k}\right)$ in the stable index list of $\varphi$, one has a uniform upper bound:

$$
\operatorname{ind}_{s t a b}(\varphi):=\sum \operatorname{ind}\left(\Phi_{k}\right) \leq N-1
$$

On the other hand, the gate index list for any train track map $f: \Gamma \rightarrow \Gamma$ is the list of the gate indices

$$
\operatorname{ind}_{\mathbf{G}(f)}\left(v_{k}\right)=\frac{g\left(v_{k}\right)}{2}-1
$$

at the essential vertices $v_{k}$ of $\Gamma$, where $g\left(v_{k}\right)$ is the number of gates at $v_{k}$, and $v_{k}$ is essential if it has at least 3 gates and is $f$-periodic. The advantage of the the gate index list over the above described index list is that it can be read off directly from the given train track map.

It turns out that Statement 3 of Theorem 1.1 is a consequence of Statements 1 and 2. This follows from standard results on Outer space and $\mathbb{R}$-trees. For the convenience of the reader we recall and assemble the relevant facts in Sections 8.

It is a direct consequence of Nielsen-Thurston theory for surface homeomorphisms that in the special case where $\varphi$ is geometric, i.e. $\varphi$ is induced 
by a homeomorphism of some surface with boundary, the above stable index inequality (1.1) becomes an equality. In general, however, computer experiments of the first author (see [6, subsection 7.3], and [4]) indicate that random automorphisms have very low stable index; indeed, for up to rank $N \leq 9$ more than half of the investigated automorphisms have index list equal to $\left[\frac{1}{2}\right]$, to $\left[\frac{1}{2}, \frac{1}{2}\right]$, to $[1]$ or to $\left[\frac{1}{2}, \frac{1}{2}, \frac{1}{2}\right]$.

In our subsequent work [6] we use Theorem 1.1 as crucial tool to produce explicit automorphisms $\varphi_{I} \in \operatorname{Out}\left(F_{N}\right)$ which realize as stable index list any given list $I=\left[j_{1}, \ldots, j_{\ell}\right]$ of positive values $j_{k} \in \frac{1}{2} \mathbb{Z}$ which satisfies the above inequality (1.1), thus answering a question posed by Handel and Mosher [11].

Theorem 1.1 is also used to derive in Corollary 6.7 information about the elements of the monoid generated by train track morphisms on a common graph with respect to a fixed gate structure. This result is also the "door opener" to a further study of strata in Outer space, in analogy to the well known and heavily investigated strata in Teichmüller space, see for instance [22].

Acknowledgements: This paper came into existence largely due to Catherine Pfaff's postdoc stay in Marseille: It is part of a larger project on which we started working with her during the last months of her position in Marseille. Although the content of our discussions mostly concern the companion paper [6] (which was indeed meant to be joint work with her), some of the material presented in this paper as well must surely be influenced by having talked to Catherine.

We would also like to thank Ilya Kapovich for some valuable comments on indices of random automorphisms, in the context of his recent work [16] with Pfaff.

\section{Notation AND CONVENTIONS}

Throughout this paper, $F_{N}$ will denote the non-abelian free group of finite rank $N \geq 2$, and $\operatorname{Out}\left(F_{N}\right)$ the group of its outer automorphisms. Furthermore, we will use the following conventions and notations:

A graph is always connected, without vertices of valence 1, and finite, unless it is the universal covering $\widetilde{\Gamma}$ of a finite connected graph $\Gamma$. For every oriented edge $e$ of $\Gamma$ we denote by $\bar{e}$ the edge with reversed orientation. The set $E^{ \pm}(\Gamma)$ denotes the set of all edges $e$ including their "inverses" $\bar{e}$, while by $E^{+}(\Gamma)$ we mean a section (sometimes called an orientation) of the quotient map $e \mapsto\{e, \bar{e}\}$ on $E^{ \pm}(\Gamma)$.

An edge path $\gamma$ in $\Gamma$ is a (possibly infinite) sequence $\gamma=\ldots e_{i-1} e_{i} e_{i+1} \ldots$ of edges $e_{i} \in E^{ \pm}(\Gamma)$ where the initial vertex of any $e_{i}$ must agree with the terminal vertex of $e_{i+1}$. Such an edge path $\gamma$ is reduced if it doesn't contain any backtracking subpath $\gamma^{\prime}$, i.e. $\gamma^{\prime}$ is a finite sub-edge-path which has coinciding initial and terminal vertex, and which is contractible relative to its endpoints. 
If a finite edge path $\gamma$ is not reduced, then it is always homotopic rel. endpoints in $\Gamma$ to a reduced edge path $[\gamma]$, and this homotopy can be expressed as iterative contraction of backtracking subpaths.

The combinatorial length $|\gamma|$ of a finite edge path $\gamma$ is equal to the number of edges traversed by $\gamma$, and $\gamma$ is trivial if $|\gamma|=0$.

A graph map is a map $f: \Gamma \rightarrow \Gamma^{\prime}$ between graphs which maps vertices to vertices and edges $e$ to (not necessarily reduced) edge paths $f(e)$. If $f$ has no contracted edges, i.e. none of the image paths $f(e)$ is trivial, then $f$ induces a differential $D f: E^{ \pm}(\Gamma) \rightarrow E^{ \pm}\left(\Gamma^{\prime}\right)$ which maps $e$ to the initial edge of $f(e)$.

Two edges $e$ and $e^{\prime}$ with same initial vertex $v$ form a turn $\left(e, e^{\prime}\right)$ at $v$, and the map $D f$ induces a map $D^{2} f:\left(e, e^{\prime}\right) \mapsto\left(D f(e), D f\left(e^{\prime}\right)\right)$ from the turns of $\Gamma$ to the turns of $\Gamma^{\prime}$. The turn $\left(e, e^{\prime}\right)$ is degenerate if we have $e=e^{\prime}$ in $E^{ \pm}(\Gamma)$.

We say that an edge path $\gamma=\ldots e_{i-1} e_{i} e_{i+1} \ldots$ crosses over (or uses) a turn $\left(e, e^{\prime}\right)$ if for some index $i$ one has $e_{i}=\bar{e}$ and $e_{i+1}=e^{\prime}$.

To every graph map $f: \Gamma \rightarrow \Gamma^{\prime}$ there is associated a non-negative transition matrix $M(f)=\left(m_{e^{\prime}, e}\right)_{e^{\prime} \in E^{+}\left(\Gamma^{\prime}\right), e \in E^{+}(\Gamma)}$, where the coefficient $m_{e^{\prime}, e}$ is the number of times that the (possibly unreduced) edge path $f(e)$ crosses over the edge $e^{\prime}$ or the inversely oriented edge $\bar{e}^{\prime}$ (both occurrences counted positively!). It follows directly from the definition that for any two graph maps $f: \Gamma \rightarrow \Gamma^{\prime}$ and $g: \Gamma^{\prime} \rightarrow \Gamma^{\prime \prime}$ one has:

$$
M(g \circ f)=M(g) M(f) .
$$

Recall that a non-negative square matrix $M$ is primitive if there is a positive power $M^{t}$ with positive coefficients only.

An automorphism $\varphi \in \operatorname{Out}\left(F_{N}\right)$ is called irreducible with irreducible powers (iwip) or fully irreducible if there is no proper free factor of $F_{N}$ which is fixed up to conjugacy by any positive power of $\varphi$.

A graph map $f: \Gamma \rightarrow \Gamma$ is called expanding if for every edge $e \in E^{ \pm}(\Gamma)$ there is an exponent $t \geq 1$ such that $\left|f^{t}(e)\right| \geq 2$. If $f$ represents an iwip automorphism, then, up to passing to a quotient graph through contracting $f$-invariant subtrees in $\Gamma$, the map $f$ must be expanding.

\section{Gate Structures FOR GRAPhS}

Definition 3.1. (1) A gate structure $\mathbf{G}_{v}$ at a vertex $v$ of a graph $\Gamma$ is a partition of the edges $e \in E^{ \pm}(\Gamma)$ with initial vertex $v$ into equivalence classes, called gates.

(2) A gate structure $\mathbf{G}$ on $\Gamma$ is the collection of gate structures $\mathbf{G}_{v}$ for every vertex $v$ of $\Gamma$. A graph $\Gamma$ together with a gate structure $\mathbf{G}$ on $\Gamma$ has been termed a train track in [20].

(3) Two edges $e, e^{\prime} \in E^{ \pm}(\Gamma)$ with same initial vertex $v$ form a legal turn $\left(e, e^{\prime}\right)$ (with respect to $\mathbf{G}$ ) if $e$ and $e^{\prime}$ belong to distinct gates. Otherwise the turn $\left(e, e^{\prime}\right)$ is called illegal. 
(4) A (finite or infinite) edge path $\gamma=\ldots e_{i-1} e_{i} e_{i+1} \ldots$ in $\Gamma$ is called legal (with respect to $\mathbf{G}$ ), if all of the turns $\left(\bar{e}_{i}, e_{i+1}\right)$ over which $\gamma$ crosses are legal.

These notions, introduced in [20] and in a similar fashion elsewhere ([1], $[10],[11], \ldots)$, have been inspired by the fundamental paper [2], where the gate structure $\mathbf{G}=\mathbf{G}(f)$ is defined through a train track map $f: \Gamma \rightarrow \Gamma$, as explained below in Definition 3.8 and Remark 3.10. There are other natural occurrences of gate structures, for example given by an edge-isometric $F_{N^{-}}$ equivariant map from the universal cover $\widetilde{\Gamma}$ to an $\mathbb{R}$-tree $T$, see [20].

Definition 3.2. Let $\Gamma$ and $\Gamma^{\prime}$ be graphs equipped with gate structures $\mathbf{G}$ and $\mathbf{G}^{\prime}$ respectively. A graph map $f: \Gamma \rightarrow \Gamma^{\prime}$ is called a train track morphism if the following two conditions hold:

(1) $f$ has no contracted edges.

(2) $f$ has the train track property: it maps legal paths to legal paths.

Remark 3.3. The reader can verify directly that condition (2) of Definition 3.2 is equivalent to the following two more local conditions:

(2'a) the map $D^{2} f$ (see Section 2) maps legal turns to legal turns, and

(2'b) for every edge $e$ of $\Gamma$ the edge path $f(e)$ is legal.

Remark 3.4. (a) It follows directly from Definition 3.2 that the composition $g \circ f$ of two train track morphisms $f: \Gamma \rightarrow \Gamma^{\prime}$ and $g: \Gamma^{\prime} \rightarrow \Gamma^{\prime \prime}$, with respect to the same gate structure on $\Gamma^{\prime}$, is again a train track morphism.

(b) In particular, in the special case $\Gamma^{\prime}=\Gamma$ and $\mathbf{G}^{\prime}=\mathbf{G}$ we note that for any edge $e$ and any integer $t \geq 0$ the edge path $f^{t}(e)$ is legal and hence reduced.

Remark 3.5. If $\Gamma$ is equipped with two gate structures $\mathbf{G}_{1}$ and $\mathbf{G}_{2}$, such that every gate of $\mathbf{G}_{2}$ is contained in a gate of $\mathbf{G}_{1}$, then $\mathbf{G}_{2}$ is finer than (or a refinement of) $\mathbf{G}_{1}$, while $\mathbf{G}_{1}$ is coarser than $\mathbf{G}_{2}$.

Any train track morphism $f: \Gamma \rightarrow \Gamma^{\prime}$ stays a train track morphism if the gate structure of $\Gamma$ is replaced by a coarser one, or the gate structure of $\Gamma^{\prime}$ by a finer one.

In order to pinpoint certain subtleties which will trouble us later, we define:

Definition 3.6. (a) Let $\Gamma$ and $\Gamma^{\prime}$ be graphs equipped with gate structures $\mathbf{G}$ and $\mathbf{G}^{\prime}$ respectively. A graph map $f: \Gamma \rightarrow \Gamma^{\prime}$ is called a gate structure morphism if the following two conditions hold:

(1) $f$ has no contracted edges.

(2) The induced map $D f$ (see Section 2) maps all edges in any gate of $\mathbf{G}$ to a common image gate of $\mathbf{G}^{\prime}$. In other words: $f$ induces a well defined map $f_{\mathbf{G}}: \mathbf{G} \rightarrow \mathbf{G}^{\prime}$.

(b) If $f$ is not necessarily a gate structure morphism on all of $\Gamma$, but induces a well defined map $f_{\mathbf{G}_{v}}: \mathbf{G}_{v} \rightarrow \mathbf{G}_{f(v)}^{\prime}$ on the gates at a given vertex $v$ of $\Gamma$, we say that $f$ is a local gate structure morphism at $v$. 
We'd like to alert the reader that the notions of "train track morphisms" and "gate structure morphisms" are sort of perpendicular to each other, as neither of them implies the other: A gate structure morphism is a train track morphism $f$ if and only if the induced map $f_{\mathbf{G}}$ is injective and the image of every edge is legal. On the other hand, a general train track morphism may be quite far from being a gate structure morphism, as the identity map which passes from given gates structure to a strict refinement (compare Remark 3.5) shows. However, for the special case of graph self-maps we have the following observation which will turn out to be often quite useful:

Lemma 3.7. Let the graph self-map $f: \Gamma \rightarrow \Gamma$ be a train track morphism with respect to some gate structure $\mathbf{G}$ on $\Gamma$.

Then at every $f$-periodic vertex $v$ of $\Gamma$ the induced map $D^{2} f$ maps legal to legal and illegal to illegal turns. In particular, $f$ induces a well defined bijective map from the gates at $v$ to the gates at $f(v)$. In other words:

For every periodic vertex $v$ the map $f$ induces a local gate structure morphism $f_{\mathbf{G}_{v}}: \mathbf{G}_{v} \rightarrow \mathbf{G}_{f(v)}$ which is bijective.

Proof. Since $f$ has the train track property, the induced map $D f$ maps edges in distinct gates to edges in distinct gates. Thus for every vertex $v$ of $\Gamma$ the number of gates at $f(v)$ must be larger or equal to the number of gates at $v$. Furthermore, if the two numbers are equal, it follows directly that edges in any given gate must be mapped by $D f$ to edges that also lie all in a common gate. This implies directly the claimed statement for periodic vertices of a train track self-morphism.

The most important special case of graph maps, and also the source of the notion of "gates", is the case of a self-map $f: \Gamma \rightarrow \Gamma$. It turns out that self-maps which don't even have the train track property define already a gate structure on $\Gamma$ :

Definition 3.8. Let $f: \Gamma \rightarrow \Gamma$ be a graph self-map with no contracted edges. The intrinsic gate structure $\mathbf{G}=\mathbf{G}(f)$ is defined by declaring edges $e, e^{\prime}$ with same initial vertex to belong to the same gate if and only if some power of $f$ maps $e$ and $e^{\prime}$ to edge paths which have non-trivial initial subpaths in common.

Remark 3.9. (1) This definition has built in that $f$ preserves the gates of the gate structure $\mathbf{G}(f)$ :

(a) edges in distinct gates at a common vertex are mapped into distinct gates and,

(b) all edges in a given gate are mapped into the same gate.

In other words: The graph self-map $f$ is a gate structure morphism with respect to $\mathbf{G}(f)$, and the induced map $f_{\mathbf{G}(f)}$ is injective. 
(2) Hence, by Remark 3.3, in order to check whether $f$ is a train track morphism with respect to $\mathbf{G}(f)$, we only need to check that for every edge $e$ the edge path $f(e)$ is legal.

Remark 3.10. (1) Recall that a classical train track map as introduced by Bestvina and Handel [2] is a graph self-map $f: \Gamma \rightarrow \Gamma$ which has the property that for any edge $e$ and any integer $t \geq 1$ the edge path $f^{t}(e)$ is reduced.

We have already noted in Remark 3.4 (b) that any train track self-morphism $f: \Gamma \rightarrow \Gamma$, with respect to any gate structure $\mathbf{G}$ on $\Gamma$, is such a classical train track map.

Conversely, it follows directly from the above definitions that every classical train track map is a train track morphism with respect to the intrinsic gate structure $\mathbf{G}(f)$.

(2) However, the reader should be warned that for any classical train track map $f$ as above, in addition to $\mathbf{G}(f)$ there may well be other gate structures $\mathbf{G}$, with respect to which $f$ is also a train track morphism: For example every positive automorphism is represented by a train track morphism on the rose with respect to the gate structure at the sole vertex which consists only of the "positive" and of the "negative" gates.

(3) On the other hand, it follows directly from the above definitions that any other such gate structure $\mathbf{G}$ must be coarser than $\mathbf{G}(f)$, so that $\mathbf{G}(f)$ is indeed the finest gate structure with respect to which the train track map $f$ is a train track morphism.

The following turns out to be a useful notion for the sequel. In order to properly state it we recall from Lemma 3.7 that any train track self-map $f$ which fixes a vertex $v$ induces a well defined bijection $f_{\mathbf{G}_{v}}$ on the set of gates at that vertex.

Definition 3.11. A train track morphism $f: \Gamma \rightarrow \Gamma$ with respect to some gate structure $\mathbf{G}$ is called gate-stable if $f$ fixes every vertex of $\Gamma$, and at every vertex $f$ fixes also every gate of $\mathbf{G}$.

We notice directly that every train track self-morphism which acts periodically on every vertex possesses a positive power that is gate-stable.

We say that a path $\gamma$ crosses over a gate turn $\left(\mathfrak{g}_{i}, \mathfrak{g}_{j}\right)$ if $\gamma$ contains the subpath $\bar{e} \cdot e^{\prime}$ with $e \in \mathfrak{g}_{i}$ and $e^{\prime} \in \mathfrak{g}_{j}$.

Definition 3.12. Let $f: \Gamma \rightarrow \Gamma$ be a train track morphism with respect to some gate structure $\mathbf{G}$ on $\Gamma$.

(a) For every vertex $v$ of $\Gamma$ we define the gate-Whitehead-graph $W h_{\mathbf{G}}^{v}(f)$ to be the graph with the set $\mathbf{G}_{v}$ of gates at $v$ as vertex set, and with a (nonoriented) edge connecting $\mathfrak{g}_{i}$ to $\mathfrak{g}_{j}$ if for some $t \geq 1$ and some edge $e \in E^{ \pm}(\Gamma)$ the path $f^{t}(e)$ crosses over $v$ entering through $\mathfrak{g}_{i}$ and leaving through $\mathfrak{g}_{j}$. 
(b) A vertex $v$ in $\Gamma$ is essential if it is periodic and if there are at least three gates at $v$. The gate index at $v$ is defined as

$$
\operatorname{ind}_{\mathbf{G}}(v):=\frac{g(v)}{2}-1
$$

where $g(v)$ denotes the number of gates at $v$.

(c) The gate index list for $f$ is the list of gate indices of $\mathbf{G}(f)$ at essential vertices. We usually order such a list as decreasing sequence of its values.

For graph self-maps the Whitehead graphs at the vertices (in various dialects) have been used previously (e.g. see $[2,11,12,14,20]$ ).

Proposition 3.13. Let $f$ and $g$ be train track morphisms of a graph $\Gamma$ with respect to the gate structure $\mathbf{G}:=\mathbf{G}(f)$. If $g$ induces an automorphism of $\pi_{1}(\Gamma)$ and is gate-stable, then for every vertex $v$ of $\Gamma$ the graph $W h_{\mathbf{G}}^{v}(f)$ is a subgraph of both, $W h_{\mathbf{G}}^{v}(f \circ g)$ and $W h_{\mathbf{G}}^{v}(g \circ f)$.

Proof. We first use the hypothesis $\mathbf{G}=\mathbf{G}(f)$ to deduce (see Remark 3.9 (1)) that $f$ is a gate structure morphism and hence induces a map $f_{\mathbf{G}}: \mathbf{G} \rightarrow \mathbf{G}$. Hence there is a well defined map $D_{\mathbf{G}}^{2} f$ on the gate turns of $\Gamma$, given by $D_{\mathbf{G}}^{2} f\left(\mathfrak{g}_{i}, \mathfrak{g}_{j}\right):=\left(f_{\mathbf{G}}\left(\mathfrak{g}_{i}\right), f_{\mathbf{G}}\left(\mathfrak{g}_{j}\right)\right)$.

For any vertex $v$ of $\Gamma$ it follows from the definition of the gate-Whiteheadgraph that in $W h_{\mathbf{G}}^{v}(f)$ two "vertices" $\mathfrak{g}_{i}$ and $\mathfrak{g}_{j}$ are connected by an edge if and only if one of the following occurs:

(a) For some edge $e \in E^{ \pm}(\Gamma)$ the path $f(e)$ crosses over the gate turn $\left(\mathfrak{g}_{i}, \mathfrak{g}_{j}\right)$.

(b) For some $t \geq 1$ the map $D_{\mathrm{G}}^{2} f^{t}$ maps one of the gate turns crossed over by some $f(e)$ to the gate turn $\left(\mathfrak{g}_{i}, \mathfrak{g}_{j}\right)$.

From the hypothesis that $g$ is a homotopy equivalence and our convention that graphs don't have valence 1 vertices we deduce that each edge $e$ appears in the image $g\left(e^{\prime}\right)$ of some edge $e^{\prime}$. It follows that all gate turns $\left(\mathfrak{g}_{i}, \mathfrak{g}_{j}\right)$ crossed over by the path $f(e)$ are also crossed over by the path $f \circ g\left(e^{\prime}\right)$. Since $g$ is gate-stable, the maps $g_{\mathbf{G}}$ and $D_{\mathbf{G}}^{2} g$ are well defined, and $D_{\mathbf{G}}^{2} g$ acts as the identity on the set of gate turns. This implies both, that $g \circ f(e)$ crosses over the same gate turns as $f(e)$, and that the above property (b) for $f$ is equivalent to property (b) for $f \circ g$ or $g \circ f$.

This shows that, for every vertex $v$ of $\Gamma$, in the graph $W h_{\mathbf{G}}^{v}(f)$ two "vertices" $\mathfrak{g}_{i}$ and $\mathfrak{g}_{j}$ are connected by an edge only if the same "vertices" are also connected by an edge in $W h_{\mathbf{G}}^{v}(f \circ g)$ and in $W h_{\mathbf{G}}^{v}(g \circ f)$.

\section{IN THE ABSENCE OF INPS}

The notion of an INP is a classical concept, going back to [2]. Originally, "INP" was an abbreviation for "indivisible Nielsen path"; however, through frequent use it has become mainly an acronym, and as such we treat it here: 
Definition 4.1. Let $f: \Gamma \rightarrow \Gamma$ be a train track morphism with respect to some gate structure $\mathbf{G}$ on $\Gamma$. A reduced path $\eta$ in $\Gamma$ is called a periodic INP if $\eta=\gamma^{-1} \circ \gamma^{\prime}$, where the two branches $\gamma$ and $\gamma^{\prime}$ are non-trvial legal paths, and $f^{t}(\eta)$ is homotopic relative endpoints to $\eta$, for some $t \geq 1$.

The reader should be alerted that the endpoints of a periodic INP $\eta$ may well not be vertices of $\Gamma$ (although this can be readily achieved by subdividing edges and their iterated $f$-images, which is a finite procedure since the endpoints of $\eta$ are by definition $f$-periodic).

The importance of the gate-Whitehead-graph (see Definition 3.12) for a train track map is underlined by the following "irreducibility criterion" from [12]. We quote here only a simplified version which is used below in Section 6:

Proposition 4.2 ([12, Proposition 5.1]). Let $f: \Gamma \rightarrow \Gamma$ be a train track representative of $\varphi \in \operatorname{Out}\left(F_{N}\right)$. Assume furthermore:

(1) The transition matrix $M(f)$ is primitive.

(2) The gate-Whitehead-graph $W h_{\mathbf{G}(f)}^{v}(f)$ for $f$ at every vertex $v$ of $\Gamma$ is connected.

(3) There is no periodic INP for $f$ in $\Gamma$.

Then $\varphi$ is iwip (= fully irreducible).

A "partial converse" of this result is given by the following:

Proposition 4.3 ([12, Proposition 5.1]). Let $f: \Gamma \rightarrow \Gamma$ be a train track representative of some iwip automorphism $\varphi \in \operatorname{Out}\left(F_{N}\right)$. Assume that $f$ is expanding and that there is no periodic INP for $f$.

Then $f$ must satisfy conditions (1) and (2) from Proposition 4.2.

Remark 4.4. Since the connectedness of the gate-Whitehead-graph for $f$ at every vertex is a direct consequence of the connectedness of the classical Whitehead graph of $f$ at $v$, one can obtain Proposition 4.3 also as direct consequence of the classical known irreducibility criterion (see [13]).

Alternatively, the reader may prefer to go for a direct proof of Proposition 4.3 according to the following lines: If $M(f)$ is not primitive, then the transition matrix $M\left(f^{t}\right)$ of a positive power of $f$ must be reducible, so that there is an $f^{t}$-invariant subgraph of $\Gamma$, which by expansiveness of $f$ must have as fundamental group a proper free factor of $F_{N}$.

Similarly, if $W h_{\mathbf{G}(f)}^{v}(f)$ is not connected for some vertex $v$ of $\Gamma$, then we can introduce "invisible edges" to blow up that vertex and thus again find, as complement of the invisible edges, an invariant subgraph with a proper free factor of $F_{N}$ as fundamental group. (More details regarding the blow-up technology of vertices by means of invisible edges can be found in the proof of [12, Proposition 5.1], or in the description before Lemma 6.6 of [14].) 


\section{LONG TURNS}

Recall that every graph map $f: \Gamma \rightarrow \Gamma^{\prime}$ which induces an isomorphism on $\pi_{1} \Gamma$ (i.e. $f$ is a homotopy equivalence) possesses a cancellation bound which can be expressed topologically as a bounded backtracking constant of any lift $\widetilde{f}: \widetilde{\Gamma} \rightarrow \widetilde{\Gamma}^{\prime}$ to the universal coverings (see [9]): There exists a constant $C(f) \geq 0$ such that for any reduced edge path $\gamma$ in $\widetilde{\Gamma}$ the unreduced image path $\widetilde{\widetilde{f}}(\gamma)$ is contained in the $C(f)$-neighborhood of the reduced path $[\widetilde{f}(\gamma)]$, for the metric on $\widetilde{\Gamma}^{\prime}$ defined by combinatorial path length (see $\S 2$ ). It is known (see $[3,9])$ for homotopy equivalences $f$ that the combinatorial volume of $f(\Gamma)$, i.e. the total length of the edge paths $f\left(e_{i}\right)$ for all $e_{i} \in \mathrm{E}^{+}(\Gamma)$, is such a cancellation bound.

Definition 5.1. Let $\Gamma$ and $\Gamma^{\prime}$ be graphs equipped with gate structures, and let $f: \Gamma \rightarrow \Gamma^{\prime}$ be a train track morphism.

(1) A long turn $\left(\gamma, \gamma^{\prime}\right)$ in $\Gamma$ is given by two non-trivial legal edge paths $\gamma$ and $\gamma^{\prime}$ with common initial vertex of $\Gamma$ and distinct first edges.

(2) A long turn $\left(\gamma, \gamma^{\prime}\right)$ is called illegal if the initial edges of $\gamma$ and $\gamma^{\prime}$ form an illegal turn (in the sense of Definition $3.1(3))$. Otherwise $\left(\gamma, \gamma^{\prime}\right)$ is called legal.

(3) A long turn $\left(\gamma_{1}, \gamma_{1}^{\prime}\right)$ in $\Gamma^{\prime}$ is called the $f$-image of the long turn $\left(\gamma, \gamma^{\prime}\right)$ in $\Gamma$, denoted by

$$
f^{L T}\left(\gamma, \gamma^{\prime}\right):=\left(\gamma_{1}, \gamma_{1}^{\prime}\right),
$$

if $f(\gamma)=\gamma_{0} \circ \gamma_{1}$ and $f\left(\gamma^{\prime}\right)=\gamma_{0}^{\prime} \circ \gamma_{1}^{\prime}$ such that $\gamma_{0}=\gamma_{0}^{\prime}$ is the maximal common initial subpath of $f(\gamma)$ and $f\left(\gamma^{\prime}\right)$, and $\gamma_{1}$ and $\gamma_{1}^{\prime}$ are non-trivial paths.

A long turn which possesses an $f$-image is called an $f$-long turn. Otherwise we call it $f$-degenerate.

Note that a turn $\left(e, e^{\prime}\right)$ in the classical sense of Definition $3.1(3)$ is in particular a long turn, except in the particular case where it is degenerate, i.e. $e=e^{\prime}$.

Remark 5.2. We note that the existence of a cancellation bound $C(f)$ for a train track morphism $f: \Gamma \rightarrow \Gamma^{\prime}$ together with the "no contracted edges" assumption in Definition 3.2 imply that every long turn $\left(\gamma, \gamma^{\prime}\right)$ with $|\gamma|,\left|\gamma^{\prime}\right| \geq$ $C(f)+1$ is $f$-long.

We now want to consider the composition $g \circ f$ of two train track morphisms $f: \Gamma \rightarrow \Gamma^{\prime}$ and $g: \Gamma^{\prime} \rightarrow \Gamma^{\prime \prime}$. We observe:

(1) If a long turn $\left(\gamma_{1}, \gamma_{2}\right)$ in $\Gamma$ is $f$-degenerate, then $f\left(\gamma_{1}\right)$ is an initial subpath of $f\left(\gamma_{2}\right)$ (or conversely), and hence, since $\gamma_{1}, \gamma_{2}$ as well as their images are legal, $g \circ f\left(\gamma_{1}\right)$ is an initial subpath of $g \circ f\left(\gamma_{2}\right)$ (or conversely), so that $\left(\gamma_{1}, \gamma_{2}\right)$ is also $(g \circ f)$-degenerate. 
(2) Similarly, if $\left(\gamma_{1}, \gamma_{2}\right)$ is $f$-long, but its $f$-image long turn $\left(\gamma_{1}^{\prime}, \gamma_{2}^{\prime}\right)$ is $g$ degenerate, it follows that $\left(\gamma_{1}, \gamma_{2}\right)$ is also $(g \circ f)$-degenerate.

(3) On the other hand, if $\left(\gamma_{1}, \gamma_{2}\right)$ is $f$-long and its $f$-image long turn $\left(\gamma_{1}^{\prime}, \gamma_{2}^{\prime}\right)$ is $g$-long, then we see directly that $\left(\gamma_{1}, \gamma_{2}\right)$ is also $(g \circ f)$-long, and that its $(g \circ f)$-image coincides with the $g$-image of $\left(\gamma_{1}^{\prime}, \gamma_{2}^{\prime}\right)$.

We summarize:

Lemma 5.3. Let $f: \Gamma \rightarrow \Gamma^{\prime}$ and $g: \Gamma^{\prime} \rightarrow \Gamma^{\prime \prime}$ be two train track morphisms (with respect to the same gate structure on $\Gamma^{\prime}$ ).

A long turn $\left(\gamma_{1}, \gamma_{2}\right)$ in $\Gamma$ is $(g \circ f)$-long if and only if $\left(\gamma_{1}, \gamma_{2}\right)$ is $f$-long and its $f$-image long turn $f^{L T}\left(\gamma_{1}, \gamma_{2}\right)$ is $g$-long. In this case one has:

$$
(g \circ f)^{L T}\left(\gamma_{1}, \gamma_{2}\right)=g^{L T}\left(f^{L T}\left(\gamma_{1}, \gamma_{2}\right)\right)
$$

Remark 5.4. If a long turn $\left(\gamma, \gamma^{\prime}\right)$ in $\Gamma$ is legal, then it is $f$-long for every train track morphism $f: \Gamma \rightarrow \Gamma^{\prime}$, and the $f$-image is again legal (indeed, the common initial subpath $\gamma_{0}=\gamma_{0}^{\prime}$ from part (3) of Definition 5.1 is in this case trivial).

On the other hand, for an illegal $f$-long turn the $f$-image can be either legal or illegal; both cases do occur.

Remark 5.5. (1) Any long turn $\left(\gamma_{1}^{\prime}, \gamma_{2}^{\prime}\right)$ in $\Gamma$ is called a subturn of a long turn $\left(\gamma_{1}, \gamma_{2}\right)$ if each $\gamma_{i}^{\prime}$ is an initial subpath of $\gamma_{i}$, for $i=1$, 2. If both long turns, $\left(\gamma_{1}, \gamma_{2}\right)$ and $\left(\gamma_{1}^{\prime}, \gamma_{2}^{\prime}\right)$, are $f$-long for some train track map $f$, then their $f$-images are either both legal or both illegal. Hence, in order to test legality of the $f$-image of any long turn, it suffices to calculate the $f$-image of the shortest $f$-long subturn of the given long turn.

(2) If the length of a long turn $\left(\gamma_{1}, \gamma_{2}\right)$, defined as $\min \left\{\left|\gamma_{1}\right|,\left|\gamma_{2}\right|\right\}$, satisfies $\min \left\{\left|\gamma_{1}\right|,\left|\gamma_{2}\right|\right\} \geq C \geq 0$, then we will denote below the subturn $\left(\gamma_{1}^{\prime}, \gamma_{2}^{\prime}\right)$ of $\left(\gamma_{1}, \gamma_{2}\right)$ with $\left|\gamma_{1}^{\prime}\right|=\left|\gamma_{2}^{\prime}\right|=C$ by:

$$
\left(\gamma_{1}, \gamma_{2}\right) \dagger^{C}:=\left(\gamma_{1}^{\prime}, \gamma_{2}^{\prime}\right)
$$

We also denote the set of all long turns $\left(\gamma_{1}, \gamma_{2}\right)$ in $\Gamma$ with branch length $\left|\gamma_{1}\right|=$ $\left|\gamma_{2}\right|=C$ by $L T_{C}(\Gamma)$.

Definition 5.6. A train track morphism $f: \Gamma \rightarrow \Gamma^{\prime}$ is called legalizing if every illegal $f$-long turn has legal $f$-image.

It follows from Remarks 5.2 and 5.5 that a train track map $f$ with cancellation bound $C(f)$ is legalizing if and only if every long turn built from legal paths of length $C(f)+1$ has legal $f$-image. Indeed, it follows from Corollary 6.5 below that many (or even "most") train track representatives of iwip automorphisms are legalizing. 
Remark 5.7. The reader should be warned that in the (frequently occurring) case that a graph map $f: \Gamma \rightarrow \Gamma^{\prime}$ is a train track morphism with respect to a gate structure $\mathbf{G}$ on $\Gamma$ and simultaneously with respect to a coarser gate structure $\mathbf{G}_{0}$, then $f$ may well be legalizing with respect to $\mathbf{G}$ but not with respect to $\mathbf{G}_{0}$.

We should perhaps point out here that although every legalizing train track morphism maps every path $\gamma$ with a single illegal turn to a legal path $[f(\gamma)]$ (after reduction!), the same does not at all follow for a path $\gamma$ with more than one illegal turn. The only conclusion one can draw is that the number of illegal turns in the reduced image path $[f(\gamma)]$ is at most half times the number of illegal turns in $\gamma$ plus 1.

This notion of a "legalizing map" is robust and easy to handle, as is illustrated by following:

Proposition 5.8. Let $f: \Gamma_{1} \rightarrow \Gamma_{2}$ and $g: \Gamma_{2} \rightarrow \Gamma_{3}$ be two train track morphisms. If either $f$ or $g$ is legalizing, then the composition $g \circ f$ is a legalizing train track morphism.

Proof. This follows directly from Lemma 5.3, together with the observation in Remark 5.4 that for any train track morphism and any legal long turn the image long turn is always legal.

The following observation will be used crucially in the next section:

Lemma 5.9. (a) Let $g: \Gamma \rightarrow \Gamma$ be a train track morphism with respect to some gate structure $\mathbf{G}$, and assume that $g$ is both, legalizing and gate-stable. Then the gate structure $\mathbf{G}$ is equal to the intrinsic gate structure of $g$ :

$$
\mathbf{G}=\mathbf{G}(g)
$$

(b) Moreover, if $f: \Gamma \rightarrow \Gamma$ is another train track morphism with respect to $\mathbf{G}$, then the intrinsic gate structure $\mathbf{G}(f \circ g)$ of the composition $f \circ g$ is equal $\mathbf{G}$.

Proof. (a) From the hypothesis that $g$ is legalizing we know that the $g$-image of every $g$-long illegal turn $\left(\gamma, \gamma^{\prime}\right)$ is a legal long turn. But since $g$ is gatestable, the initial edges of $g(\gamma)$ and $g\left(\gamma^{\prime}\right)$ must lie in the same gate, so that they cannot form a legal turn. Hence they must belong to the common initial subpath of $g(\gamma)$ and $g\left(\gamma^{\prime}\right)$ and thus indeed be identical.

Since this is true for any illegal turn, all legal paths exiting from the same gate must have $g$-images with coinciding initial edge. This proves that the gate structure $\mathbf{G}$ is finer than or equal to $\mathbf{G}(g)$. The converse is true for any self-map $g$ that is a train track morphism with respect to a given gate structure G, see Remark 3.10 (3).

(b) Since $f$ is a train track morphism and hence has no contracted edges, the above proved fact, that $D g$ maps all edges in any given gate to a single edge, 
is inherited by $D(f \circ g)$. Hence the arguments from the previous paragraph are also true for $f \circ g$ instead of $g$, so that we obtain $\mathbf{G}(f \circ g)=\mathbf{G}$.

Remark 5.10. (1) Note that the proof of the last lemma stays valid if the hypothesis " $g$ gate-stable" is replaced by the weaker assumption " $g$ gate structure morphism" (see Definition 3.6). In particular, by Lemma 3.7 it suffices to assume that all vertices of $\Gamma$ are periodic under the map $g$.

(2) Note also that there is a delicacy in Statement (b) of the last lemma: The analogous statement for the composition $g \circ f$ is in general wrong, unless one assumes that $f$ is a gate structure morphism.

\section{LEGALIZING MAPS FOR IWIP AUTOMORPHISMS}

We will now concentrate on the situation of a classical train track map $f: \Gamma \rightarrow \Gamma$, which is a train track morphism with respect to the intrinsic gate structure $\mathbf{G}(f)$ on $\Gamma$, see Remark 3.10. We assume furthermore that $f$ is a homotopy equivalence so that it possesses a cancellation bound $C(f)$, and that it satisfies the following expansion property:

Definition 6.1. For any constant $K \geq 1$ a train track morphism $f: \Gamma \rightarrow \Gamma^{\prime}$ is called strongly $K$-expanding if every legal edge path $\gamma$ in $\Gamma$ of length $|\gamma| \geq K$ has $f$-image which is strictly longer:

$$
|f(\gamma)| \geq|\gamma|+1
$$

Remark 6.2. Expanding train track morphisms (see §2) are not necessarily strongly $K$-expanding for some $K \geq 1$ (and conversely), but it follows directly from the definitions that every expanding train track morphism has a positive power which is strongly 1-expanding.

We define the minimal stretching factor $\lambda_{\min }^{K}(f)$ of $f$ for legal paths of length $\geq K$ by:

$$
\lambda_{\min }^{K}(f):=\min \left\{\frac{|f(\gamma)|}{|\gamma|} \mid \gamma \text { legal of length }|\gamma| \geq K\right\}
$$

We will now derive from any cancellation bound $C(f) \geq 0$ of a strongly $K$-expanding train track morphism $f: \Gamma \rightarrow \Gamma$ an expansion bound $C(f)^{+} \geq 0$ :

Lemma 6.3. Let $f: \Gamma \rightarrow \Gamma$ be a train track map which possesses a cancellation bound $C(f)$ and is strongly $K$-expanding for some $K \geq 1$. We define:

$$
C(f)^{+}:=\max \left(K, \frac{C(f)}{\lambda_{\min }^{K}(f)-1}\right)
$$

Let $C \geq C(f)^{+}$be an integer. Then the map $f$ induces a map

$$
f^{L T_{C}}: L T_{C}(\Gamma) \rightarrow L T_{C}(\Gamma),\left(\gamma, \gamma^{\prime}\right) \mapsto f^{L T}\left(\gamma, \gamma^{\prime}\right) \dagger^{C} .
$$


Proof. From the definition of the minimal stretching factor $\lambda_{\min }^{K}(f)$ it follows that every legal path $\gamma$ of length $|\gamma| \geq C(f)^{+} \geq K$ is mapped by $f$ to a legal path of length $|f(\gamma)| \geq|\gamma|+C(f)$. Hence it follows from the definition of a cancellation bound $C(f)$ that any long turn $\left(\gamma, \gamma^{\prime}\right) \in L T_{C}(\Gamma)$ is $f$-long, and that its image long turn $f^{L T}\left(\gamma, \gamma^{\prime}\right)$ has length $\geq C$. Thus setting $\left(\gamma, \gamma^{\prime}\right) \mapsto$ $f^{L T_{C}}\left(\gamma, \gamma^{\prime}\right) \dagger^{C}$ defines indeed a well defined map $f^{L T_{C}}: L T_{C}(\Gamma) \rightarrow L T_{C}(\Gamma)$.

Proposition 6.4. Let $f: \Gamma \rightarrow \Gamma$ be a train track map which possesses a cancellation bound $C(f)$ and is strongly $K$-expanding for some $K \geq 1$. Let $C \geq C(f)^{+}$.

Then for any $f^{L T_{C}}$-periodic illegal long turn $\left(\gamma, \gamma^{\prime}\right) \in L T_{C}(\Gamma)$ the concatenation $\bar{\gamma} \circ \gamma^{\prime}$ contains a periodic INP as subpath. Conversely, every periodic INP in $\Gamma$ can be prolonged on both sides so that the two legal branches give an $f^{L T_{C}}$-periodic illegal long turn in $L T_{C}(\Gamma)$.

Proof. We know from Lemma 6.3 that $f$ induces a well defined map $f^{L T_{C}}$ on the long turns in $L T_{C}(\Gamma)$. Assume now that for some integer $t \geq 1$ the long turn $\left(\gamma, \gamma^{\prime}\right) \in L T_{C}(\Gamma)$ is illegal and fixed by $\left(f^{L T_{C}}\right)^{t}$. Then $\gamma$ is a subpath of $f^{t}(\gamma)$, and $\gamma^{\prime}$ is a subpath of $f^{t}\left(\gamma^{\prime}\right)$. Thus on both legal paths $\gamma$ and $\gamma^{\prime}$ there must be a fixed point, which by the illegality of the turn must be different from the initial vertex of both, $\gamma$ and $\gamma^{\prime}$. We can define $\eta$ to be the path crossing over the illegal turn and connecting those two fixed points. Then $\left[f^{t}(\eta)\right]=\eta$, and since $\eta$ crosses over precisely one illegal turn, it follows that it is a periodic INP.

Conversely, it follows from a standard calculation that the legal branches of any periodic INP $\eta$ can not be longer than $C(f)^{+}$. Thus they can be prolonged by legal paths so that this prolongation gives a long turn $\left(\gamma, \gamma^{\prime}\right) \in L T_{C}(\Gamma)$. Let now the integer $t \geq 1$ be such that $\left[f^{t}(\eta)\right]=\eta$. We consider the iterates of $\left(\gamma, \gamma^{\prime}\right)$ under $f^{t}$ and note that they all contain $\eta$ as subpath, in such a way that the illegal turn on $\eta$ coincides with the illegal turn formed by $\left(\gamma, \gamma^{\prime}\right)$ (and thus also by all of its $f^{t}$-iterates). Since $L T_{C}(\Gamma)$ is finite, eventually some such iterate $f^{k t}\left(\gamma, \gamma^{\prime}\right)$ will be $\left(f^{L T_{C}}\right)^{t}$-periodic. This shows the "converse" direction of the claim.

Corollary 6.5. Let $f: \Gamma \rightarrow \Gamma$ be an expanding train track map that represents an automorphism of $F_{N}$. Then precisely one of the following is true:

(a) The map $f$ possesses a periodic INP, or

(b) every sufficiently high power of $f$ is legalizing for $\mathbf{G}(f)$.

Proof. Since $f$ represents an automorphism, it possesses a cancellation bound. Since $f$ is expanding, any sufficiently large power of $f$ will be strongly $K$ expanding for $K=1$ (see Remark 6.2). Thus Proposition 6.4 applies, so that, in case that $f$ does not possess a periodic INP, we can deduce that there is no illegal $f$-periodic long turn $\left(\gamma, \gamma^{\prime}\right) \in L T_{C}(\Gamma)$, for $C$ as in Proposition 6.4. 
It follows that after applying $f$ iteratively at least $k_{0}:=\operatorname{card} L T_{C}(\Gamma)$ times, any long turn $\left(\gamma, \gamma^{\prime}\right) \in L T_{C}(\Gamma)$ must have become legal. Since it stays legal under further iteration of $f$ (see Remark 5.4), every $f^{k}$ with $k \geq k_{0}$ must be legalizing.

Clearly both (a) and (b) can not hold simultaneously. Thus we have proved the desired dichotomy.

One can derive from the last proof that the lower bound for the exponent of $f$ needed in statement (b) of Corollary 6.5 can be efficiently calculated from the train track map $f$ with not much effort. It turns out that it only depends on the cancellation bound $C(f)$ and on the rank $N$ of the free group $F_{N}$.

Proof of Theorem 1.1. Since $g$ is assumed to be a train track morphism with respect to the gate structure $\mathbf{G}(f)$, then so must be $f \circ g$. Since $g$ induces an automorphism on $\pi_{1} \Gamma$, so does $f \circ g$, so that $f \circ g$ is a train track morphism which represents an automorphism of $F_{N}$.

Recall from Section 2 that the transition matrix of $f \circ g$ is obtained as product $M(f \circ g)=M(f) \cdot M(g)$. Hence $M(f \circ g)$ inherits positivity from the assumed positivity of $M(f)$. Thus in particular $M(f \circ g)$ is primitive.

As $g$ is gate-stable and legalizing for $\mathbf{G}(f)$, by Lemma 5.9 the intrinsic gate structure $\mathbf{G}(f \circ g)$ is equal to $\mathbf{G}(f)$.

By hypothesis, $f$ represents an iwip automorphism. From Proposition 4.3, we know that for any vertex $v$ the graph $W h_{\mathbf{G}}^{v}(f)$ is connected. As $g$ is gatestable, by Proposition 3.13 the gate-Whitehead-graph $W h_{\mathbf{G}}^{v}(f \circ g)$ must also be connected.

We now observe from Proposition 5.8 that the composed map $f \circ g$ must be legalizing, which implies by Corollary 6.5 that there are no periodic INPs for $f \circ g$.

Thus the conditions (1), (2) and (3) of Proposition 4.2 are all satisfied for the map $f \circ g$, which hence must induce an iwip automorphism. Corollary 8.5 concludes the proof.

Remark 6.6. (1) We see from the above proof that the hypotheses in Theorem 1.1 can be weakened somewhat: In the proof it is never used that the automorphism represented by $f$ is iwip. It suffices to assume that $M(f)$ is positive, and that the gate-Whitehead-graph at every periodic vertex is connected.

(2) We also don't use the fact that the gate structure on $\Gamma$ is equal to $\mathbf{G}(f)$. It suffices to assume that $f$ and $g$ are train track morphisms with respect to some fixed gate structure $\mathbf{G}$, if in statement (3) of Theorem 1.1 the list of gate indices at the $f$-periodic vertices of $\Gamma$ is computed with respect to the gate structure G (see Definition 3.12). This is a consequence of Lemma 5.9.

(3) A careful analysis of the above proof and its various ingredients, shows that the statement of Theorem 1.1 is valid as well for the map $g \circ f$ in place 
of $f \circ g$, if one assumes in addition that $f$ is a gate structure morphism (see Remark $5.10(2))$.

We'd like to remark here that part (2) of the previous remark gives the possibility to produce, from a given train track map $f$ with a fine gate structure, through properly choosing the legalizing "perturbation map" $g$, a variety of train track maps $f \circ g$ with coarser gate structures and thus, via part (3) of Theorem 1.1, with smaller index lists than $f$. A useful technology for the deliberate production of such perturbation maps is described in the next section.

We conclude this section by passing to a larger set of product maps:

Corollary 6.7. Let $\Gamma$ be a graph equipped with a gate structure $\mathbf{G}$, and for any index $i$ of some index set I let $f_{i}: \Gamma \rightarrow \Gamma$ be a train track morphism with respect to $\mathbf{G}$. Assume that each $f_{i}$ satisfies the following properties:

(1) The intrinsic gate structure satisfies $\mathbf{G}\left(f_{i}\right)=\mathbf{G}$.

(2) The transition matrix $M\left(f_{i}\right)$ is positive.

(3) For any vertex $v$ of $\Gamma$ the graph $W h_{\mathbf{G}}^{v}\left(f_{i}\right)$ is connected.

(4) There is no periodic INP for $f_{i}$ in $\Gamma$.

(5) The map $f_{i}$ is gate stable.

Then there exist exponents $m_{i} \geq 1$ such that the properties (2) - (5) hold for every element in the monoid generated by the $f_{i}^{m_{i}}$, i.e. for any product

$$
f=f_{i_{1}}^{m_{i_{1}}} f_{i_{2}}^{m_{i_{2}}} \cdots f_{i_{s}}^{m_{i_{s}}}
$$

of the $f_{i}^{m_{i}}$ (but not their inverses!). Furthermore, any such map $f$ represents an iwip automorphism, the map $f$ is legalizing, and the index list of $f$ is equal to the list of gate indices of $\mathbf{G}$ at the vertices of $\Gamma$ with 3 or more gates.

Proof. By Corollary 6.5 there exist exponents $m_{i} \geq 1$ such that each of the maps $f_{i}^{m_{i}}$ is legalizing. Furthermore, conditions (2), (3) and (4) ensure via Proposition 4.2 that $f_{i}$ represents an iwip automorphism of $F_{N}$. Conditions (2), (3) and (5) are inherited by products, if they are satisfied by every factor. The same is true for the property "legalizing", which implies condition (4). By Lemma 5.9 condition (1) is a consequence of condition (5) together with the property "legalizing".

Hence all conditions for the factors $f$ and $g$ in Theorem 1.1 are satisfied for any of the maps $f_{i}^{m_{i}}$ as well as for any product $f$ as above. Thus the conclusion (3) of Theorem 1.1 hold as well for $f$, which proves the last assertion in the statement of Corollary 6.7.

The above corollary admits a natural extension to a more involved situation, where one considers simultaneously several graphs $\Gamma_{k}$ with gate structures $\mathbf{G}_{k}$, as well as maps $f_{i}: \Gamma_{k} \rightarrow \Gamma_{k^{\prime}}$ which induce bijections on the vertices with 3 or more gates, as well as bijections on the set of their adjacent gates. This leads one directly to consider "strata" in Outer space, in analogy to 
strata in Teichmüller space as defined by fixing the indices of the singularities of quadratic differentials, see [22].

\section{LEGALIZING FACTORY}

In this section we reduce the construction of a legalizing train track morphism to the construction of a family of "elementary" train track morphisms that each legalizes only a single illegal turn.

Proposition 7.1. Let $\Gamma$ be a graph equipped with a gate structure G. Assume that there exists an integer $L \geq 1$ which satisfies:

(1) For each illegal long turn $t=\left(\gamma, \gamma^{\prime}\right)$ of branch length $L$ there exists a train track morphism $g_{t}: \Gamma \rightarrow \Gamma$ such that $t$ is $g_{t}$-long and mapped by $g_{t}^{L T}$ to a legal long turn.

(2) There exists a train track morphism $h: \Gamma \rightarrow \Gamma$ which is strongly $K$-expanding for some $K \geq 1$.

We assume furthermore that each of the above maps $g_{t}$ and $h$ has a cancellation bound $C\left(g_{t}\right)$ or $C(h)$ respectively (which is true if they induce automorphisms of $\pi_{1}(\Gamma)$ ). Then there exists a legalizing train track morphism $g: \Gamma \rightarrow \Gamma$ which is obtained as a composition of the $g_{t}$ and $h$.

Proof. For each of the illegal long turns $t$ of $\mathbf{G}$ with branch length $L$ set $g_{t}^{\prime}:=h \circ g_{t}$, and observe that $g_{t}^{\prime}$ is strongly $K$-expanding and inherits a cancellation bound $C\left(g_{t}^{\prime}\right)$ from $h$ and $g_{t}$. Moreover, as $h$ is a train track morphism, it maps legal turns to legal turns, so that by hypothesis (1) the long turn $t$ is $g_{t}^{\prime}$-long and mapped by $g_{t}^{\prime}$ to a legal long turn.

Let $C$ be the maximum of $L$ and of all the constants $C\left(g_{t}^{\prime}\right)^{+}$, as defined in Lemma 6.3 for any of the maps $g_{t}^{\prime}$ via the cancellation bounds $C\left(g_{t}^{\prime}\right)$ and the above constant $K$. Then each $g_{t}^{\prime}$ induces a well defined map $g_{t}^{\prime L T_{C}}$ on the set of long turns $L T_{C}(\Gamma)$.

We can now build iteratively the legalizing train track morphisms we are looking for: Let $g_{0}$ be the identity map and $L T_{C}^{i l l}\left(g_{0}\right) \subset L T_{C}(\Gamma)$ be the finite set of illegal long turns in $\Gamma$ of branch length $C$. We define iteratively graph maps $g_{k}: \Gamma \rightarrow \Gamma$ and nested subsets

$$
L T_{C}^{i l l}\left(g_{k}\right) \varsubsetneqq L T_{C}^{i l l}\left(g_{k-1}\right) \varsubsetneqq \ldots \varsubsetneqq L T_{C}^{i l l}\left(g_{0}\right)
$$

by considering any turn $t^{*}$ in $L T_{C}^{\text {ill }}\left(g_{k}\right)$. From the iterative definition of $L T_{C}^{i l l}\left(g_{k}\right)$ it follows that $t^{*}$ is mapped by $g_{k}^{L T_{C}}$ to long a turn (of branch length $C$ ) which is illegal. Let $t$ be the subturn of $g_{k}^{L T_{C}}\left(t^{*}\right)$ of branch length $L \leq C$, which is of course also illegal. We set $g_{k+1}:=g_{t}^{\prime} \circ g_{k}$, and define $L T_{C}^{i l l}\left(g_{k+1}\right)$ to be the set of illegal long turns in $\Gamma$ of branch length $C$ that are mapped by $g_{k+1}^{L T_{C}}$ to an illegal long turn. Note that $g_{t}^{\prime}$ was defined so that $t^{*}$ is mapped by $g_{k+1}^{L T}$ to a long turn that contains the legal long turn $g_{t}^{L T}(t)$ as subturn and is therefore legal. Recall that if a long turn is mapped by $g_{k}^{L T_{C}}$ 
to a legal long turn, then, as $g_{t}^{\prime}$ is a train track morphism, it is also mapped by $g_{k+1}^{L T_{C}}$ to a legal long turn. In other words $L T_{C}^{i l l}\left(g_{k+1}\right) \varsubsetneqq L T_{C}^{i l l}\left(g_{k}\right)$.

From the finiteness of $L T_{C}^{i l l}\left(g_{0}\right)$ we deduce that after finitely many steps one gets $g_{n}$ with $L T_{C}^{i l l}\left(g_{n}\right)=\emptyset$, which is equivalent to stating that $g=g_{n}$ is legalizing.

\section{StABlE INDICES, BRANCHING INDICES AND GATE INDICES}

The content of this section is well known to the experts, or in close proximity of well known facts; we assemble them here for the convenience of the reader. We will use some standard tools from $\mathbb{R}$-trees and Outer space technology. For background and terminology the reader may consult [20]; further detail can be found in [7] or [23]. We follow here mostly the original source [9].

For every expanding train track map $f: \Gamma \rightarrow \Gamma$ there exists a non-negative real eigenvector $\vec{v}$ of the transition matrix $M(f)$ which has real eigenvalue $\lambda>1$, and any such $\vec{v}$ determines an $\mathbb{R}$-tree $T=T(\vec{v})$ (which in some cases is called the forward limit tree and can be considered as boundary point of Outer space $\mathrm{CV}_{N}$ ).

The tree $T=T(\vec{v})$ is obtained by choosing an arbitrary lift $\tilde{f}: \widetilde{\Gamma} \rightarrow \widetilde{\Gamma}$ of the train track map $f$ to the universal covering $\widetilde{\Gamma}$, and by defining $T$ to be the metric space associated to the pseudo-metric $d_{\infty}$ on $\widetilde{\Gamma}$ which is the limit for $t \rightarrow$ $\infty$ of the decreasing sequence of pseudo-metrics $d_{t}(x, y):=\frac{1}{\lambda^{t}} d_{\vec{v}}\left(\widetilde{f}^{t}(x), \widetilde{f}^{t}(y)\right)$. Here the pseudo-metric $d_{\vec{v}}$ on $\widetilde{\Gamma}$ is defined through lifting the $\vec{v}$-edge-lengths of $\Gamma$ that are explicitly given by the coefficients of the eigenvector $\vec{v}$.

As a consequence one obtains a canonical $F_{N}$-equivariant map $i: \widetilde{\Gamma} \rightarrow T$ which is edge-isometric with respect to the pseudo-metric $d_{\vec{v}}$, i.e. every edge $e$ of $\widetilde{\Gamma}$ is mapped by $i$ isometrically to its image $i(e) \subset T$. The map $\widetilde{f}$ also induces directly a homothety $H: T \rightarrow T$ with stretching factor $\lambda$, and one obtains the following "commutative diagram":

$$
H \circ i=i \circ \tilde{f}
$$

The map $i$ maps legal paths in $\widetilde{\Gamma}$ isometrically to segments in $T$. On the other hand, any path $\widetilde{\eta}$ in $\widetilde{\Gamma}$ which is the lift of a periodic INP in $\Gamma$ is folded by $i$ completely to a single segment, which is the isometric image of any of the two legal branches of $\widetilde{\eta}$.

It follows from standard train track arguments (see for example Section 3 of [14]) that for any path $\gamma$ in $\Gamma$ a sufficiently high $f$-iterate $f^{t}(\gamma)$ is homotopic rel. endpoints to a pseudo-legal path, i.e. a legal concatenation of legal paths and periodic INPs. It follows that for any two points $x, y \in \widetilde{\Gamma}$ one has $i(x)=i(y)$ if and only if after iterating $\widetilde{f}$ sufficiently many times the geodesic path $\widetilde{\gamma}$ in the tree $\widetilde{\Gamma}$ which joins $\tilde{f}^{t}(x)$ to $\tilde{f}^{t}(y)$ is a legal concatenation of 
legal subpaths and lifts of periodic INPs, where the legal subpaths only use edges with $d_{\vec{v}}$-length 0 . In particular, we see that the absence of INPs for $f$ implies directly that the $F_{N}$-action on $T$ is free, if all the exponents of the the eigenvector $\vec{v}$ are positive. The latter is known if the transition matrix $M(f)$ is primitive, and hence always true if the expanding train track map $f$ represents an iwip automorphism. Furthermore, the North-South result of the $\varphi$-action on the closure of $\mathrm{CV}_{N}$ proved in [17] yields:

Proposition 8.1. For any iwip automorphism $\varphi$ the forward limit tree $T=$ $T(\vec{v})$ is well defined up to uniform rescaling of the metric, and in particular does not depend on the expanding train track representative $f: \Gamma \rightarrow \Gamma$ and its primitive transition matrix $M(f)$ with Perron-Frobenius eigenvector $\vec{v}$.

If $\Gamma$ doesn't contain any non-trivial loop which is a legal concatenation of periodic INPs, then the $F_{N}$-action on $T$ by isometries is free. This conclusion is in particular true if there is no periodic INP in $\Gamma$.

As a direct consequence of the above described construction of $T$ from $\widetilde{\Gamma}$ by means of the eigenvector $\vec{v}$ one has the following fact, which is well known to the experts (see [9], [11], [20], or, for much detail, § 7 of [15]). Recall that a direction at a point $P \in T$ is a connected component of $T \backslash\{P\}$.

Proposition 8.2. Let $f: \Gamma \rightarrow \Gamma$ be an expanding train track map, and let $\vec{v}$ be an eigenvector of $M(f)$ with eigenvalue $\lambda>1$. Assume that $\vec{v}$ has only positive coefficients, and let $T=T(\vec{v})$ the corresponding forward limit tree.

(1) If there is no periodic INP for $f$, then the map i restricts to an $F_{N}$ equivariant bijection $i_{V}$ between essential vertices $v_{k}$ of $\widetilde{\Gamma}$ (i.e. lifts of $f$ periodic vertices of $\Gamma$ with 3 or more gates) on one hand, and branch points $i\left(v_{k}\right)$ of $T$ on the other. This bijection extends to a canonical bijection between the gates at any $v_{k}$ and the directions at $i\left(v_{k}\right)$ (where a gate $\mathfrak{g}_{j}$ is mapped to the direction that contains the open segments $i\left(\stackrel{\circ}{e}_{i}\right)$ for any edge $e_{i}$ in $\left.\mathfrak{g}_{j}\right)$.

(2) [Not used in the sequel.] If $f$ possesses periodic INPs, then the endpoints (assumed to have been made into vertices) of any such periodic INP $\eta$ have to be considered as equivalent, and for these endpoints one has to identify those two gates which contain the two branches of $\eta$. Then we get the precisely analogous statement as in the "no INP" case (1), except that the preimage of a branch point in $T$ will now be the lift of an $f$-periodic equivalence class of vertices in $\Gamma$ with (after the above identification) 3 or more gates.

If $X$ is a topological space, provided with a left action of a group $G$ by homeomorphisms, we say that a map $F: X \rightarrow X$ represents an automorphism $\Phi \in \operatorname{Aut}(G)$ if for all $x \in X$ and all $g \in G$ one has:

$$
\Phi(g) \cdot F(x)=F(g \cdot x)
$$

This applies in particular to the special case where $X$ is the universal covering of the quotient space $X / G$ with $\pi_{1}(X)=G$, and $F$ induces a homeomorphism 
$f: X / G \rightarrow X / G$. In this case, if $F$ represents $\Phi$, then $f$ induces on $\pi_{1}(X)=G$ the outer automorphism $\varphi$ defined by $\Phi$.

It follows from the equality $(8.1)$ and the $F_{N}$-equivariance of the map $i$ that any lift $\tilde{f}$ of the train track map $f$ represents the same automorphism $\Phi \in \operatorname{Aut}\left(F_{N}\right)$ as the associated homothety $H: T \rightarrow T$, where $\Phi$ induces (by a the previous paragraph) the outer automorphims $\varphi$ that is represented by the train track map $f$. Since the stretching factor of $H$ satisfies $\lambda>1$, it follows that $H$ has precisely one fixed point $Q$ which is either contained in $T$, or it lies in the metric completion $\bar{T}$ of $T$ (where we use the canonical extension of $H$ to $\bar{T})$.

Let now $\Phi^{\prime} \in \operatorname{Aut}\left(F_{N}\right)$ be a second lift of $\varphi$, and assume that $\Phi^{\prime}$ is isogredient to $\Phi$, i.e. $\Phi^{\prime}=\iota_{w} \circ \Phi \circ \iota_{w^{-1}}=\iota_{w} \circ \iota_{\Phi(w)^{-1}} \circ \Phi$ for some $w \in F_{N}$, where $\iota_{v}: F_{N} \rightarrow F_{N}$ denotes the conjugation $u \mapsto v u v^{-1}$. Assume furthermore that the lift $\widetilde{f}^{\prime}: \widetilde{\Gamma} \rightarrow \widetilde{\Gamma}$ of $f$ and the homothety $H^{\prime}: T \rightarrow T$ both represent the automorphism $\Phi^{\prime}$. Then we obtain $\widetilde{f}^{\prime}=w \widetilde{f} w^{-1}$ and $H^{\prime}=w H w^{-1}$, and thus deduce for the fixed point $Q^{\prime}$ of $H^{\prime}$ the equality $Q^{\prime}=w Q$.

Conversely, if $H^{\prime}=u H$ is the homothety of $T$ which represent some lift $\Phi^{\prime}=i_{u} \Phi$ of $\varphi$, then, if $H^{\prime}(Q)=Q$, the action of $u$ on $T$ must fix the point $Q$. Thus, if the $F_{N}$-action on $T$ is free, then one deduces $u=1$.

As a consequence one gets a natural injective map from the isogredience classes of lifts $\Phi_{k}$ of $\varphi$ into the set of $F_{N}$-orbits of points in $\bar{T}$, given by the fixed point $Q_{k}$ of the associated homothety $H_{k}: T \rightarrow T$ that represents $\Phi_{k}$.

In [9, Theorem 2.1 (3) and Proposition 4.4], the following has been proved (we only cite the easy case where $\operatorname{Stab}(Q)$ is trivial):

Proposition 8.3. Let $\varphi \in \operatorname{Out}\left(F_{N}\right)$ be an iwip automorphism, and let $T=$ $T(\vec{v})$ be the forward limit tree of $\varphi$, given as above by some eigenvector $\vec{v}$ with eigenvalue $\lambda>1$ of the transition matrix $M(f)$ of a train track representative $f: \Gamma \rightarrow \Gamma$ of $\varphi$. We assume that there is no periodic INP in $\Gamma$, so that the $F_{N}$-action on $T$ is free.

Let $H: T \rightarrow T$ be the homothety (with stretching factor $\lambda$ ) that represents some lift $\Phi \in \operatorname{Aut}\left(F_{N}\right)$ of $\varphi$, and assume $\Phi$ has index $\operatorname{Ind}(\Phi)>0$. Then the fixed point $Q$ of $H$ is contained in $T$, and $Q$ is a branch point of $T$. There is a natural injection $i_{Q}$ from the set of attracting fixed points of $\Phi$ on $\partial F_{N}$ to the set of directions at $Q \in T$. The image of $i_{Q}$ is precisely the set of those directions that are fixed by $H$.

From the last sentence of this proposition we see that replacing $\varphi$ (and hence also $\Phi$ and $H$ ) by a positive power will increase the image set of the map $i_{Q}$. From Gaboriau and Levitt [8] finiteness result, one knows that for any $T$ with free $F_{N}$-action every branch point has only finitely many directions, so that a suitable positive power of $H$ will indeed fix every direction of $T$ at $Q$. 
The same finiteness result [8] also implies that there are only finitely many $F_{N}$-orbits of branch points in $T$. Thus through possibly replacing $\varphi$ by a further positive power we can assume that the associated homothety $H$ of $T$ fixes every $F_{N}$-orbit of branch points of $T$. Thus for any branch point $Q^{\prime}$ of $T$ there is a suitable element $u \in F_{N}$ such that the homothety $H^{\prime}=u H$ has $Q^{\prime}$ as fixed point (pick $u$ such that $H\left(Q^{\prime}\right)=u^{-1} Q^{\prime}$ ). Now we "perturb" $\Phi$ and $\tilde{f}$ correspondingly to obtain $\Phi^{\prime}=\iota_{u} \circ \Phi$ and $\widetilde{f}^{\prime}=u \tilde{f}$, and, if need be, we pass to another common positive power, so that the homothety $H^{\prime}$ fixes every direction of $T$ at $Q^{\prime}$. As a consequence, the map $i_{Q^{\prime}}$ from Proposition 8.3 becomes a bijection between the attracting fixed points of $\Phi^{\prime}$ on $\partial F_{N}$ and the directions of $T$ at $Q^{\prime}$.

Thus we obtain:

Proposition 8.4. Let $\varphi \in \operatorname{Out}\left(F_{N}\right)$ be an iwip automorphism that has an expanding train track representative $f: \Gamma \rightarrow \Gamma$ without periodic INPs, and let $T$ be its forward limit tree.

Then, for some integer $t \geq 1$, there exists a natural bijection between on one hand the isogredience classes of representatives $\Phi_{k}$ of $\varphi^{t}$ which satisfy ind $\Phi_{k} \geq \frac{1}{2}$, and on the other hand the $F_{N}$-orbits $F_{N} \cdot Q_{k}$ of branch points $Q_{k}$ of $T$.

This correspondence extends further to a bijection between the set of attractors for the induced $\Phi_{k}$-action on $\partial F_{N}$, and the set of directions of $T$ at $Q_{k}$.

The assertion remains valid if the integer $t$ is replaced by any positive integer $t^{\prime}=k t \in \mathbb{N}$.

Recall from the Introduction that the stable index list of an automorphism $\varphi \in \operatorname{Out}\left(F_{N}\right)$ without non-trivial periodic conjugacy classes is given by the maximal (decreasing) list of indices $\operatorname{ind}\left(\Phi_{k}\right)=\frac{a\left(\Phi_{k}\right)}{2}-1$ for representatives $\Phi_{k} \in \operatorname{Aut}\left(F_{N}\right)$ of a suitable positive power of $\varphi$ that are pairwise non-isogredient, where $a\left(\Phi_{k}\right)$ denotes the number of attractors of $\Phi_{k}$ on $\partial F_{N}$.

Thus Proposition 8.4 shows that for an iwip automorphism $\varphi$, assumed to have an expanding train track representative $f: \Gamma \rightarrow \Gamma$ without periodic INPs, the stable index list of $\varphi$ agrees with the branching index list of $T$, i.e. the maximal (decreasing) sequence of values $\operatorname{ind}\left(Q_{k}\right):=\frac{b\left(Q_{k}\right)}{2}-1$ for branch points $Q_{k}$ in distinct $F_{N}$-orbits, where $b\left(Q_{k}\right)$ denotes the number of directions of $T$ at $Q_{k}$. On the other hand, we obtain directly from part (1) of Proposition 8.2 that the branching index list of $T$ agrees with the gate index list for the map $f: \Gamma \rightarrow \Gamma$, as defined in Definition 3.12. Thus we obtain:

Corollary 8.5. Let $\varphi \in \operatorname{Out}\left(F_{N}\right)$ be an iwip automorphism, and let $f: \Gamma \rightarrow \Gamma$ be an expanding train track map which represents $\varphi$ and which doesn't have any periodic INP. 
Then the stable index list of the automorphism $\varphi$ agrees with the gate index list of the map $f$.

In fact, we see from the details of the above correspondences that, after raising $\varphi$ and $f$ to a suitable common positive power, there is a natural bijection between the essential vertices of $f$ and the isogredience classes of $\varphi$, which for any essential vertex $v_{k}$ and any representative $\Phi_{k}$ of the corresponding isogredience class extends further to a bijection between the attracting fixed points of $\Phi_{k}$ on $\partial F_{N}$ and the gates at $v_{k}$. This last bijection can be seen concretely by considering the unique eigenray $\rho$ defined by any gate at $v_{k}$, its lift to an eigenray $\widetilde{\rho}$ of a (suitably chosen) lift $\widetilde{f}_{k}$ of $f$ for the universal covering $\widetilde{\Gamma}$, and the image $i(\widetilde{\rho}) \in T$, which is an eigenray of the associated homothety $H_{k}$ which represents $\Phi_{k}$. Under the canonical $F_{N}$-equivariant identification $\partial F_{N}=\partial \widetilde{\Gamma}$ the ray $\widetilde{\rho}$ represents an attractor of the $\Phi_{k}$-action on $\partial F_{N}$, and conversely, every attractor for $\Phi_{k}$ comes from such an eigenray.

\section{REFERENCES}

[1] M. Bestvina and M. Feighn, Hyperbolicity of the complex of free factors. arXiv: 1211.1730

[2] M. Bestvina, and M. Handel, Train tracks and automorphisms of free groups. Ann. of Math. (2) 135 (1992), no. 1, 1-51

[3] M. Bestvina, M. Feighn, and M. Handel, Laminations, trees, and irreducible automorphisms of free groups. Geom. Funct. Anal. 7 (1997), no. 2, 215-244

[4] T. Coulbois, Experimental results about indices of free group automorphisms. In preparation

[5] T. Coulbois, A. Hilion, Botany of irreducible automorphisms of free groups. Pacific J. Math. 256 (2012), no. 2, 291-307

[6] T. Coulbois and M. Lustig Index realization for automorphisms of free groups. Preprint 2015

[7] M. Culler, K. Vogtmann, Moduli of graphs and automorphisms of free groups. Invent. Math. 84 (1986), no. 1, 91-119

[8] D. Gaboriau and G. Levitt, The rank of actions on $\mathbb{R}$-trees. Ann. Sc. ENS 28 (1995), 549-570

[9] D. Gaboriau, A. Jaeger, G. Levitt, and M. Lustig, An index for counting fixed points of automorphisms of free groups. Duke Math. J. 93 (1998), no. 3, 425-452

[10] U. Hamenstädt, The boundary of the free splitting graph and the free factor graph. arXiv:1211.1630v4

[11] M. Handel and L. Mosher, Axes in Outer Space. Memoirs AMS 1004, Amer Mathematical Society, 2011

[12] A. Jäger and M. Lustig, Free group automorphisms with many fixed points at infinity. The Zieschang Gedenkschrift, 321-333, Geom. Topol. Monogr., 14, Geom. Topol. Publ., Coventry, 2008

[13] I. Kapovich, Algorithmic detectability of iwip automorphisms. arXiv:1209.3732

[14] I. Kapovich and M. Lustig, Invariant laminations for irreducible automorphisms of free groups. Quaterly J. Math, to appear; arXiv:1104.1265

[15] I. Kapovich and M. Lustig, Invariant laminations for irreducible automorphisms of free groups. arXiv:1104.1265v3 
[16] I. Kapovich and C. Pfaff, A train track directed random walk on Out $\left(F_{r}\right)$. arXiv:1409.8044

[17] G. Levitt and M. Lustig, Irreducible automorphisms of $F_{n}$ have North-South dynamics on compactified outer space. J. Inst. Math. Jussieu 2 (2003), no. 1, $59-72$

[18] G. Levitt and M. Lustig, Automorphisms of free groups have asymptotically periodic dynamics. J. reine u. angew. Math. 619 (2008), 1-36

[19] J. Los and M. Lustig, The set of train track representatives of an irreducible free group automorphism is contractible. CRM-Barcelona Preprint Series 2004, No 606

[20] M. Lustig, Conjugacy and centralizers for iwip automorphisms of free groups. In "Geometric Group Theory", Trends in Mathematics, 197-224. Birkhäuser Verlag, Basel, 2007

[21] M. Lustig, Folding lines in outer space determine the dual lamination of their limit $\mathbb{R}$-tree. MPI-Bonn Preprint Series 2010, No 98

[22] H. Masur and J. Smillie, Quadratic differentials with prescribed singularities and pseudo-Anosov diffeomorphisms. Comment. Math. Helvetici 68 (1993), 289-307

[23] K. Vogtmann, Automorphisms of Free Groups and Outer Space. Geometriae Dedicata 94 (2002), 1-31

Institut de mathématiques de Marseille, Université d'Aix-Marseille, 39, Rue Frédéric Joliot Curie, 13453 Marseille Cedex 13, France, thierry.coulbois@ UNIV-AMU.FR, MARTIN.LUSTIG@UNIV-AMU.FR 